\title{
Dosificación para elaborar bloques huecos de hormigón que cumplan con la actual NTE INEN 3066
}

\section{Dosage to make hollow concrete blocks that comply with the current NTE INEN 3066}

\author{
Mercedes Villacís Troncoso \\ FICA -EPN \\ Quito, Ecuador \\ mercedes.villacis@epn.edu.ec
}

\author{
Germán Luna Hermosa \\ FICA -EPN \\ Quito, Ecuador \\ german.luna@epn.edu.ec
}

\author{
Erick Velastegui Zambrano \\ FICA -EPN \\ Quito, Ecuador \\ erick-velastegui@hotmail.com
}

\author{
Wilmer Adrián Santacruz \\ FICA -EPN \\ Quito, Ecuador \\ santacruz.wil.adn@gmail.com
}

\author{
Paúl Zuñiga Morales \\ FICA -EPN \\ Quito, Ecuador \\ paul.zunigam@epn.edu.ec
}

\author{
James David Nates Pasaje \\ FICA -EPN \\ Quito, Ecuador \\ james.nates@epn.edu.ec
}

\author{
Liseth Orbe Pinchao \\ FICA -EPN \\ Quito, Ecuador \\ liseth.orbep@epn.edu.ec
}

\begin{abstract}
Resumen-En Ecuador la industria dedicada a la elaboración de bloques huecos de hormigón no cuenta con un método de dosificación, cuyos procesos de producción se realizan empíricamente, utilizando agregados de peso ligero como pómez pura o pómez con arena (chasqui), y se combina con polvo (arena fina de baja densidad y de color blanquecino); estos son materiales con propiedades diferentes a los convencionales que exige la norma para bloques de hormigón NTE INEN-3066. Lo que explica, en gran parte, por qué los bloques que se elaboran en el país no alcanzan las resistencias mínimas exigidas por dicha normativa. La metodología mostrada en el ACI 211.2 de hormigón estructural liviano se considera la más aplicable para este tipo de material que implica realizar varias mezclas de prueba las cuales, mediante un análisis estadístico, permiten generar una relación agua-cemento correcta. Este tipo de dosificación obtiene una optimización en las proporciones de agregado liviano que mejoran el comportamiento de los bloques huecos, alcanzando un incremento de un $70 \%$ en su resistencia, en relación con los bloques artesanales. El resultado de la presente investigación es una tabla de dosificación para las tres clases de bloques que puedan utilizar los artesanos para garantizar el producto que fabrican.
\end{abstract}

Abstract - In Ecuador, the industry dedicated to the manufacture of hollow concrete blocks does not have a dosage method, so production processes are carried out empirically, using lightweight aggregates such as pure pumice or pumice with sand (chasqui), and combined with powder, which is a fine, low density, off-white sand; these materials have different properties to those conventional required by the Ecuadorian standard for concrete blocks, NTE INEN-3066. This largely explains why the blocks that are made in this country do not reach the minimum strengths required by these norms. The methodology shown in ACI 211.2 for lightweight structural concrete is considered the most applicable for this type of material; it implies carrying out several test mixtures which, through statistical analysis, allow generating a correct water-cement ratio. This type of dosage achieves optimization in the proportions of light aggregate that improves the behavior of the hollow blocks, performing an increase of $70 \%$ in its resistance in relation to artisan blocks. The result of this investigation is a dosage table for the three types of blocks, which can be used by artisans to guarantee the quality of the product they manufacture.

Sumario: I Introducción, II Metodología, III Resultados y Discusión, IV Conclusiones.

Como citar: Villacís, Mercedes., Luna, Germán., Velastegui, Erick., Santacruz, Wilmer., Zúñiga, Paúl., Nates, James., Orbe, Liseth. (2020). Dosificación para elaborar bloques huecos de hormigón que cumplan con la actual NTE INEN 3066. Revista Tecnológica - Espol, 32(1). Recuperado a partir de http://www.rte.espol.edu.ec/index.php/tecnologica/article/view/688 


\section{INTRODUCCIÓN}

En abril de 2016 Ecuador vivió un terremoto de $7.8 \mathrm{Mw}$, según la escala sismológica de Richter, dejando grandes daños en infraestructura, perjuicios económicos, pero sobre todo la irreparable pérdida de vidas humanas. Este hecho recordó la importancia de contar con un alto nivel de calidad no solo de sistemas constructivos, sino también, de los materiales que se utilizan [1].

En respuesta a este hecho, las exigencias de las propiedades mecánicas para las unidades de mampostería se pusieron a la altura del estándar que demanda las normas ASTM en Estados Unidos y que son referencia para la mayoría de los controles de calidad en la construcción; sin embargo, tal actualización, en materia de parámetros de calidad, no consideró el proceso productivo de la industria local [8]. Así, por ejemplo, al no existir un método de diseño de mezclas para la elaboración de bloques, se usan los criterios del ACI 211.1 en el que se dosifica utilizando ripio, arena, cemento y agua que cumplan con determinadas condiciones normativas, pero, estos materiales son diferentes a los que se emplean en Ecuador para fabricar bloques huecos de hormigón [1], [3], [4], [5].

La problemática se presenta en el momento en que las bloqueras que existen en el país fabrican los mampuestos con cemento portland, agua y utilizan como agregados materiales livianos no normalizado como la piedra pómez. En este estudio se utilizó la piedra pómez con dos tamaños, conocidos como Chasqui grueso y Chasqui fino, así como el polvo que es una arena fina de la zona, los cuales no cumplen con la normativa exigida por el Instituto Ecuatoriano de Normalización [2].

Esta clase de agregados influye directamente en las propiedades del mampuesto provocando un alto grado de absorción y capacidad limitada de soportar cargas, pero, a su vez, generan unidades livianas de alta resistencia al fuego, buen aislamiento térmico y acústico.

El intentar aprovechar estos beneficios en el mampuesto resulta limitado, dado el escaso uso de hormigón ligero en el Ecuador y la falta de información respecto de las características de los materiales de peso liviano, con una consecuente metodología empírica de dosificación en la que se varía la proporción de los materiales con base en la experiencia obtenida durante años, dando como resultado el no poder asegurar la capacidad mecánica real del mampuesto, y centrar el diseño únicamente en el costo y las dimensiones solicitadas por el mercado.

Así se explica, en parte, por qué los bloques que se elaboran en el país no alcanzan las resistencias mínimas establecidas en las normativas. Por ejemplo, el mínimo valor de resistencia a compresión que deben cumplir los bloques para ser usados en mampostería estructural es 13.8 MPa (Tipo A), en mampostería no estructural es $4.0 \mathrm{MPa}$ (Tipo B) y en alivianamientos de losas $1.7 \mathrm{MPa}$ (Tipo C). Según la información obtenida en un análisis sobre una base de datos de 600 ensayos realizados en el laboratorio LEMSUR-EPN se demuestra que la resistencia alcanzada de los bloques artesanales es en promedio de 2.1 MPa, resultando un producto sobrecalificado para alivianamiento, pero insuficiente para mamposterías, es decir, presenta un desperdicio de recursos [2].
El presente caso de estudio fue realizado con materiales y procesos de fabricación de la provincia de Cotopaxi, que constituye uno de los mercados de bloques huecos de hormigón más grandes del país, que abastece a todas las provincias.

Por lo antes mencionado, el presente proyecto tiene como objeto evaluar las propiedades físicas y mecánicas de los materiales livianos no convencionales, a fin de desarrollar dosificaciones para obtener mampuestos competentes en los que se aprovechen las ventajas que proporciona el agregado liviano. Asimismo, el documento presenta observaciones al proceso actual de fabricación para que se pueda optimizar cada fase con base a las experiencias de esta investigación.

\section{METODOLOGÍA}

Según la normativa vigente, NTE INEN-3066, un bloque se define como una pieza prefabricada con forma de paralelepípedo, con o sin perforaciones en su interior, elaborada con hormigón simple, conformado por áridos inertes tanto gruesos como finos, cemento hidráulico y agua, con o sin aditivos [2].

$\mathrm{Al}$ ser prefabricados su manipulación debe ser fácil, su peso generalmente no sobrepasa los $15 \mathrm{~kg}$; en tal virtud, ya que el agregado fino es el componente que mayor volumen ocupa en la mezcla (entre el $40-60 \%$ ) se opta por la utilización de agregados de peso liviano; puesto que su densidad definirá el peso del producto final.

A lo largo de los años, las dosificaciones empíricas que utiliza la industria informal para la elaboración de bloques artesanales no toman en cuenta las características físicas granulometría, densidades aparentes, peso específico, absorción, contenido orgánico y módulo de finura - de agregados livianos como el chasqui y el polvo [6].

A esto se suma que la formación académica de los profesionales está orientada a lograr dosificaciones fundamentadas en Metodología ACI 211.1, que como se ha mencionado sus rangos de aceptación se han establecido para materiales convencionales, y no considera agregados livianos como lo que se usan en la producción de bloques artesanales.

En este sentido, establecer una alternativa de dosificación teniendo en cuenta las propiedades intrínsecas de los materiales utilizados, más allá de ser un aporte teórico, es una forma de complementar el conocimiento y brindar un soporte técnico para quienes se interesen en aplicar esta nueva metodología de dosificación en el futuro.

Por lo expuesto, la metodología que se desarrollará a continuación se fundamenta en obtener unidades de concreto que, con la mínima densidad, logren la mayor resistencia y a su vez que el contenido de cemento empleado sea el menor, a fin de reducir costos de producción.

\section{A. Identificación de los materiales que se emplean para la elaboración de bloques huecos en Ecuador.}

Durante la fase de estudio de campo y levantamiento de información se determinó que junto con el agua y el cemento se utilizan tres agregados livianos para la 
fabricación de bloques: chasqui grueso, chasqui fino y polvo.

1) Chasqui grueso: Es una roca de origen volcánico generalmente de color blanco amarillento. Se origina cuando la lava se proyecta al aire y ésta sufre una gran descompresión, dejando escapar gases y generando una textura rugosa con pequeñas celdas interconectadas. Por tal formación, la roca adquiere alta porosidad $y$ en consecuencia gran capacidad de absorción.

Este tipo de piedra pómez toma el nombre de "chasqui" por la zona de la cual se lo obtiene - minas ubicadas en el sector El Chasqui al lado oeste del volcán Cotopaxi - y por su tamaño se le adiciona el término "grueso" ya que en estado natural el tamaño de los granos puede variar desde $4.75 \mathrm{~mm}$ hasta $25 \mathrm{~mm}$.

2) Chasqui fino: También de origen volcánico, este tipo de piedra pómez se extrae de la misma zona; pero a diferencia del material anterior su tamaño no supera los $9.5 \mathrm{~mm}$

3) Polvo: Denominado así por su color blanco, textura fina y liviano, es el agregado de menor tamaño nominal de los tres que se utilizan para la fabricación de bloques. Se lo extrae de minas ubicadas en grandes montañas cercanas a las zonas pobladas entre Latacunga y Pujilí, razón por la cual las bloqueras artesanales prefieren asentarse en sitios aledaños a los mencionados.

Tras la experimentación se evidenció que este material no aporta ninguna propiedad mecánica relevante al bloque, sino que por su finura otorga un acabado cerrado a la unidad.

4) Cemento: El cemento utilizado para la fabricación de bloques se prevé que sea de alta resistencia inicial.

5) Agua: El agua debe ser potable.

\section{B. Descripción del proceso actual de fabricación de bloque hueco artesanal.}

1) Dosificación: Al evaluar la dosificación que usa la industria artesanal, cuyas cantidades de agregados se controlan en unidades de volumen, se determinó lo descrito en la TABLA I.

TABLA I

DOSIFICACIÓN QUE USA LA INDUSTRIA ARTESANAL

\begin{tabular}{|c|c|c|}
\hline Descripción & Volumen $\mathbf{( d m 3 )}$ & Dosificación aprox. \\
\hline Chasqui Grueso & 93.7 & 11.2 \\
\hline Chasqui Fino & 140.5 & 16.8 \\
\hline Polvo & 187.34 & 22.4 \\
\hline Agua & 54.6 & 6.5 \\
\hline Cemento & 8.4 & 1.0 \\
\hline
\end{tabular}

En volumen la proporción de los tres agregados (Polvo: Chasqui Fino: Chasqui Grueso) es 1 : $0.75: 0.5$; proporción a la que se restringió la dosificación del estudio.

2) Mezcla: Los agregados se combinan en humedad natural en una mezcladora mecánica, se mezclan inicialmente con una parte de agua por al menos 5 minutos permitiendo su saturación. Finalmente, se añade el cemento y el agua de mezclado restante.

La cantidad de agua para la mezcla no está limitada a la necesaria para la saturación del agregado o para satisfacer una relación a/c definida, sino a la que permita una buena trabajabilidad de la mezcla, sin que la pasta se segregue por debajo del molde y que, además, facilite desmoldar el bloque sin fisurarlo.

Por tanto, esta etapa empírica finaliza cuando la consistencia de la mezcla es la adecuada para continuar con la siguiente fase que es el formado en la máquina vibrocompactadora.

3) Moldeo: Obtenida la mezcla, se la coloca sobre la máquina vibro-compactadora permitiendo el llenado de los moldes. A continuación, se deja caer el apisonador y se espera alrededor de 10 segundos para desmoldar los bloques. En esta instancia se verifica la presencia de segregación o fisuración, por exceso o falta de agua, respectivamente.

4) Secado: Una vez desmoldados los bloques, durante las primeras horas se colocan sobre tableros a cielo abierto precautelando la acción directa de rayos solares para evitar fisuras por contracción. Debido a estas condiciones, los fabricantes optan por trabajar durante la madrugada.

5) Curado: Transcurridas 2-3 horas de haber desmoldado los bloques se inicia el curado, generalmente con una manguera que permita humedecer la superficie de los bloques procurando evitar que la aplicación de la presión de agua los fisure.

\section{Requisitos de la normativa ecuatoriana para bloques huecos de hormigón}

La normativa vigente, NTE INEN-3066 presenta los requisitos mínimos, tanto físicos como mecánicos, que deben cumplir los bloques huecos de hormigón, que se muestran de la siguiente manera:

- Dimensiones en la TABLA II

- Absorción y densidad en la TABLA III

- Resistencia a la compresión neta en la TABLA IV.

TABLA II

ESPESORES MÍNIMOS DE PAREDES Y TABIQUES EN BLOQUE TIPO A

\begin{tabular}{|c|c|c|c|}
\hline $\begin{array}{c}\text { Ancho } \\
\text { modular } \\
(\mathbf{m m})\end{array}$ & $\begin{array}{c}\text { Paredes } \\
\mathbf{( m m )}\end{array}$ & $\begin{array}{c}\text { Tabiques } \\
\mathbf{( m m )}\end{array}$ & $\begin{array}{c}\text { Área mínima de } \\
\text { tabiques } \\
(\mathbf{m m} \mathbf{m} \mathbf{m} \mathbf{2})\end{array}$ \\
\hline$<100$ & 19 & 19 & 45140 \\
\hline $101-150$ & 25 & 19 & 45140 \\
\hline$>50$ & 32 & 19 & 45140 \\
\hline
\end{tabular}

TABLA III ABSORCIÓN EN BLOQUE TIPO A

\begin{tabular}{|l|c|c|c|}
\hline \multicolumn{1}{|c|}{ Tipo } & $\begin{array}{c}\text { Densidad } \\
\mathbf{( k g / m 3 )}\end{array}$ & $\begin{array}{c}\text { Absorción } \\
\text { máxima } \\
\text { promedio } \\
(\mathbf{k g} / \mathbf{m} 3)\end{array}$ & $\begin{array}{c}\text { Absorción } \\
\text { máxima por } \\
\text { unidad (kg/m3) }\end{array}$ \\
\hline Liviano & $<1680$ & 288 & 320 \\
\hline Medio & $1680-2000$ & 240 & 272 \\
\hline Normal & $>2000$ & 208 & 240 \\
\hline
\end{tabular}

TABLA IV

RESISTENCIA A LA COMPRESIÓN NETA $\left(\mathrm{kg} / \mathrm{cm}^{2}\right)$

\begin{tabular}{|c|c|c|c|}
\hline Número de bloques & Clase A & Clase B & Clase C \\
\hline Promedio de 3 unidades & 140.76 & 40.8 & 17.34 \\
\hline 1 unidad & 126.48 & 35.7 & 14.28 \\
\hline
\end{tabular}

Con base en el cumplimiento de los requisitos antes detallados, la norma delimita el uso que pueden tener los mampuestos según su clasificación y se indica en la TABLA V. 
TABLA V

CLASIFICACIÓN POR USO

\begin{tabular}{|c|c|}
\hline Clase & Uso \\
\hline A & Mampostería estructural \\
\hline B & Mampostería no estructural \\
\hline C & Alivianamiento en losas \\
\hline
\end{tabular}

D. Determinación de las propiedades físicas y mecánicas del bloque hueco artesanal

Mediante ensayos de laboratorio llevados a cabo, según los procedimientos que se detallan en los anexos de la normativa NTE INEN-3066, se evaluaron las propiedades del bloque artesanal, obteniendo los resultados que se exponen en TABLA VI.

TABLA VI

PROPIEDADES DEL BLOQUE ARTESANAL

\begin{tabular}{|c|c|c|}
\hline Propiedad & Valor & Clasificación \\
\hline Resistencia a la compresión & $33.44 \mathrm{~kg} / \mathrm{cm} 2$ & Tipo C \\
\hline Absorción & $299.1 \mathrm{~kg} / \mathrm{m} 3$ & $>288$ \\
\hline Densidad & $1376.9 \mathrm{~kg} / \mathrm{m} 3$ & Liviano \\
\hline
\end{tabular}

El contenido de cemento para este bloque fue: 59.3 $\mathrm{kg} / \mathrm{m}^{3}$ de agregados.

E. Determinación de la dosificación para bloques huecos de hormigón que cumplan con la Norma NTE INEN 3066 (Fase experimental)

La parte experimental inició con la caracterización de los tres agregados livianos. [1]

Se ensayaron agregados de seis fuentes distintas, dentro de la zona de estudio, y se determinó que sus resultados tienen un rango de variación bajo.

Como punto de partida para determinar la dosificación, se utilizó la metodología ACI 211.2 (Metodología para determinar dosificaciones para hormigón estructural liviano), por tanto, se evaluaron las propiedades separando la fracción gruesa de la fina. [2]

Para establecer la relación óptima de fracción fina y gruesa, se probaron distintas proporciones y mediante ensayos de compresión se definió la mejor en términos de resistencia.

- Proporciones: (Chasqui Fino: Chasqui Grueso: Polvo)

- Proporción \#1; $0.75: 0.50: 1.00$

- Proporción \#2; $0.50: 0.50: 1.00$

- Proporción \#3; $1.00: 0.50: 1.00$

- Proporción \#4; $1.00: 1.00: 1.00$

- Proporción \#5; $0.75: 0.00: 1.00$

El contenido de cemento para este bloque fue: 59.3 $\mathrm{kg} / \mathrm{m}^{3}$ de agregados.

Los resultados de los ensayos a compresión para las diferentes proporciones se muestran en Fig. 1.

Como se evidencia en la Fig. 1 la Proporción \#3 refleja los mejores resultados en términos de resistencia. Adicional a esto, la experimentación permitió determinar que: al aumentar el chasqui fino, y al disminuir el chasqui grueso, la resistencia aumenta; no obstante, es imposible prescindir del chasqui grueso, puesto que, al eliminarlo la resistencia baja considerablemente.

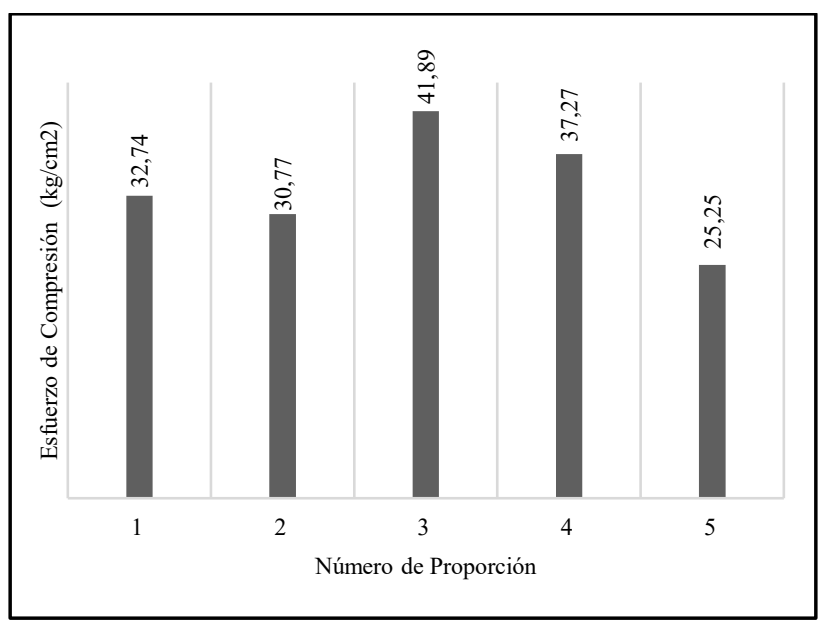

Fig. 1 Evaluación de resistencia en bloques obtenidos a partir de diferentes proporciones de agregados

En la Fig. 2 se obtiene la curva ajustada para los distintos contenidos de cemento por metro cúbico de agregado, en función de la resistencia alcanzada, evidenciándose que no responde a la tendencia lineal como lo sugiere el ACI 211.2.

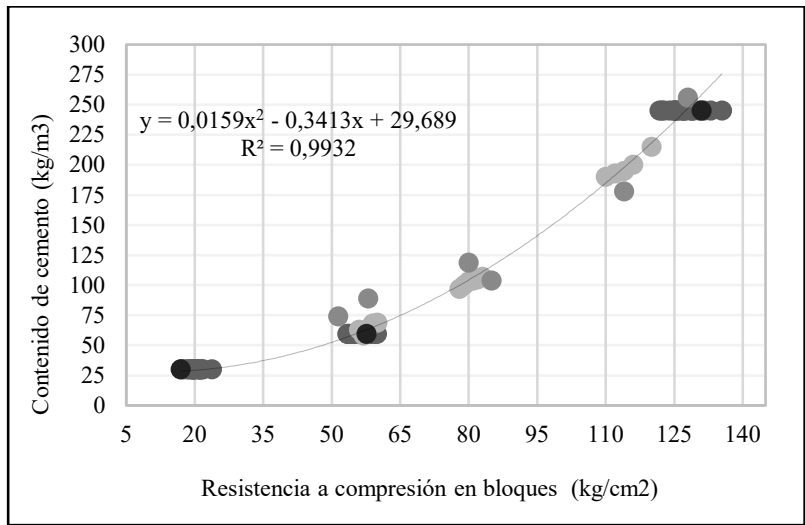

Fig. 2 Contenido de cemento en kg por metro cúbico de agregado, en función de la resistencia obtenida mediante ensayos de compresión en bloques huecos.

La forma de la curva polinómica de grado dos se ajusta de buena manera a los resultados experimentales. En la parte izquierda, se evidencia que un pequeño aumento de cemento genera un incremento considerable de resistencia, mientras que la parte derecha se observa que un aumento considerable de cemento es necesario para tener incrementos en la resistencia del bloque.

Adicional a la correlación entre la cantidad de cemento por metro cúbico de agregados y la resistencia obtenida, se encontraron límites de trabajabilidad de la mezcla en cuanto a la cantidad de cemento. El límite inferior de trabajabilidad se determinó en $30 \mathrm{~kg}$ de cemento por cada metro cúbico de agregados, pues un contenido menor genera bloques muy frágiles de manipular en las fases siguientes al moldeo, pese a que en este contenido ya se logra la resistencia necesaria para clasificar como bloque tipo $\mathrm{C}$, es aún muy frágil.

Por otra parte, el límite superior se establece en $245 \mathrm{~kg}$ de cemento por metro cúbico de agregado; ya que, un contenido de cemento mayor genera mezclas muy 
pegajosas que no permiten el llenado, vibrado y, sobre todo, imposibilitan desmoldar el bloque sin dañarlo.

A partir de la curva generada, Fig. 2, se determinó el contenido de cemento necesario para lograr las resistencias que permitan calificar a un bloque como A, B y C, lo que se muestra en la TABLA VII.

TABLA VII

ESTIMACIONES DE CANTIDAD DE CEMENTO POR METRO CÚBICO DE AGREGADO PARA CADA TIPO DE BLOQUE

\begin{tabular}{|c|c|c|}
\hline TIPO DE BLOQUE & $\begin{array}{c}\text { CONTENIDO } \\
\text { DE CEMENTO } \\
\text { POR METRO } \\
\text { CÚBICO DE } \\
\text { AGREGADO }\end{array}$ & OBSERVACIÓN \\
\hline C- Alivianamiento & $28.55 \mathrm{~kg} / \mathrm{m} 3$ & $\begin{array}{l}\text { Resistencia de } 17.34 \\
\mathrm{~kg} / \mathrm{cm} 2\end{array}$ \\
\hline B-Pared no estructural & $42.23 \mathrm{~kg} / \mathrm{m} 3$ & $\begin{array}{l}\text { Resistencia de } 42.23 \\
\mathrm{~kg} / \mathrm{cm} 2\end{array}$ \\
\hline A-Pared estructural & $296.68 \mathrm{~kg} / \mathrm{m} 3$ & $\begin{array}{ll}\text { Contenido } & \text { de } \\
\text { cemento } & \text { no } \\
\text { trabajable } & \\
\end{array}$ \\
\hline A-Pared estructural & $240.88 \mathrm{~kg} / \mathrm{m} 3$ & $\begin{array}{lr}\text { Solo cumple la } \\
\text { Resistencia por } \\
\text { bloque, mas no el } \\
\text { promedio de } 3 \\
\text { unidades }\end{array}$ \\
\hline
\end{tabular}

\section{RESULTADOS Y DISCUSIÓN}

\section{A. Análisis de resultados}

La TABLA VIII muestra la dosificación final a utilizarse en cuatro resistencias a compresión diferentes, para máquina mezcladora con capacidad de medio saco de cemento (Rendimiento aproximado: 60-70 bloques).

Con las dosificaciones establecidas, se elaboraron los distintos tipos de bloque y se evaluaron propiedades como densidad y absorción.

En la Fig. 3 y Fig. 4 se muestra la variación de la cantidad de cemento y de la absorción, por tipo de bloque respectivamente.

En la Fig. 5 y Fig. 6 se muestra la variación de la resistencia a la compresión simple y la densidad por tipo de bloque respectivamente.

TABLA VIII

DOSIFICACIÓN FINAL PARA CUATRO RESISTENCIAS A COMPRESIÓN

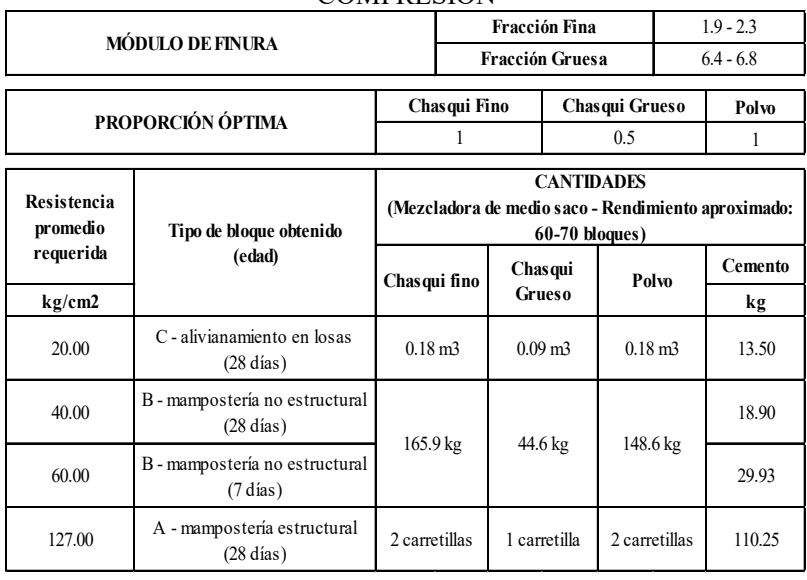

En la Fig. 3 y Fig. 4 se evidencia que la absorción es inversamente proporcional a la cantidad de cemento.

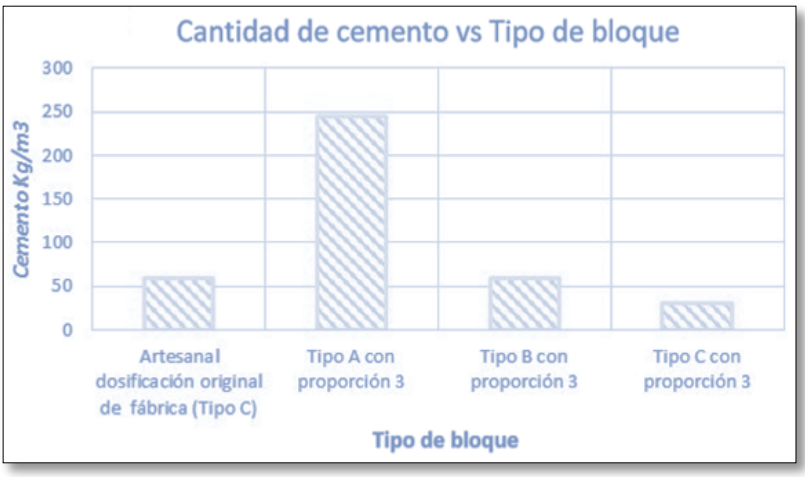

Fig. 3 Variación de la cantidad de cemento por tipo de bloque

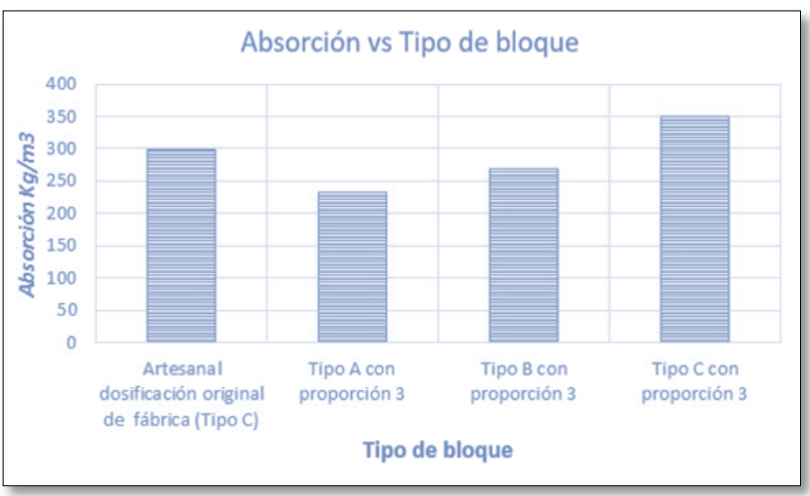

Fig. 4 Variación de la absorción por tipo de bloque

Se puede observar en la Fig. 5 y Fig. 6, que la resistencia está directamente relacionada a la densidad; a menor densidad, menor resistencia a compresión simple.

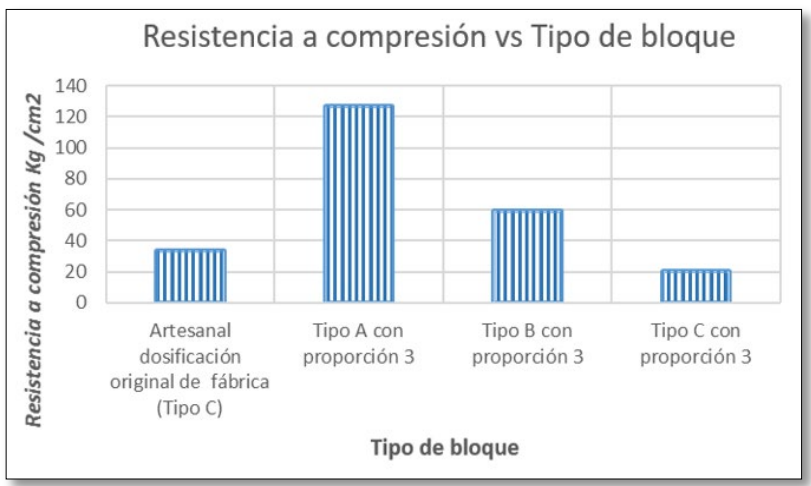

Fig. 5 Variación de la resistencia a compresión simple por tipo de bloque

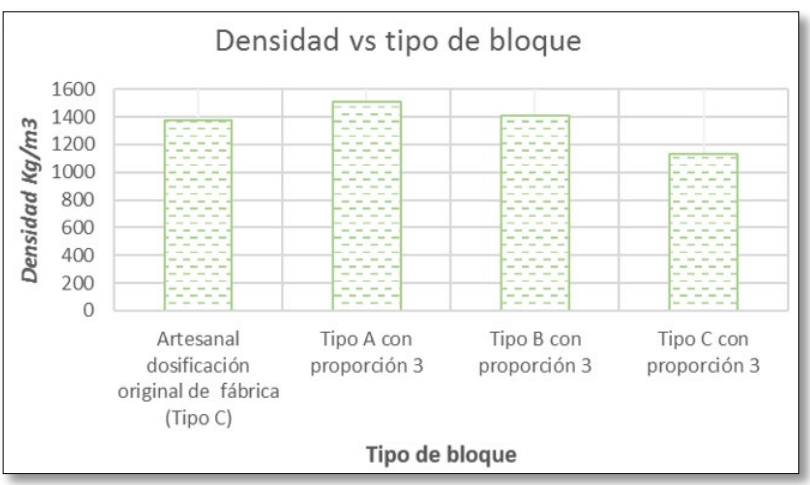

Fig. 6 Variación de la densidad por tipo de bloque 


\section{B. Análisis técnico - económico}

El análisis económico permitió establecer los costos de producción actual de una bloquera artesanal y los que se obtendrían con la aplicación de las dosificaciones aplicando la metodología propuesta.

Una vez determinados los costos a través del análisis de precios unitarios se evaluó el beneficio en términos de la resistencia o clasificación obtenida por bloque.

En la TABLA IX se presenta los costos directos obtenidos para cada tipo de bloque.

TABLA IX

COSTOS DIRECTOS POR TIPO DE BLOQUE

\begin{tabular}{|c|c|}
\hline Tipo de bloque & $\begin{array}{c}\text { Costo directo } \\
\text { (USD) }\end{array}$ \\
\hline Artesanal (Dosificación de fábrica) Tipo C & 0.1543 \\
\hline Tipo A con proporción óptima & 0.3513 \\
\hline Tipo B con proporción óptima & 0.1544 \\
\hline Tipo C con proporción óptima & 0.1258 \\
\hline
\end{tabular}

\section{CONCLUSIONES}

\section{A. Respecto a los agregados.}

La bibliografía recomienda emplear materiales de fracción fina con módulos de finura entre 3.5 y 4.2, en el caso de estudio, los tres materiales que conforman tanto la fracción gruesa como la fina, tienen módulos de finura cercanos a 6.5 y 2 respectivamente, esto no permite que el material encaje en su totalidad dentro de los límites granulométricos que establece la normativa de agregado ligero.

Se logró determinar las propiedades físicas y mecánicas de los 3 agregados livianos (Chasqui fino, chasqui grueso y polvo), obteniendo un rango del módulo de finura (TABLA VIII), tanto de la fracción fina como de la fracción gruesa, que es aplicable a las minas que abastecen a las fábricas de bloques artesanales en Cotopaxi.

Se determinó que el polvo no presenta ninguna propiedad que aporte al incremento de resistencia, sin embargo, permite que las muestras adquieran una textura adecuada.

En el agregado de peso ligero las partículas de menor tamaño tienen densidades mayores a las partículas de mayor tamaño, debido al incremento en la porosidad de estas últimas.

Las granulometrías de los materiales analizados por separado no cumplen con los límites establecidos para el agregado ligero, a menos que se mezclen en alguna proporción conveniente. Sin embargo, la proporción $\mathrm{N}^{\circ} 3$ presentó el mayor valor de resistencia pese a no encajar perfectamente en los límites granulométricos de la fracción fina y gruesa. Adicionalmente, al incrementar o disminuir la proporción se presenta una disminución de la resistencia o un mal acabado, respectivamente.

El incremento de chasqui grueso disminuye la resistencia, sin embargo, no se lo puede eliminar totalmente pues la resistencia baja considerablemente lo que se verifica con la proporción $\mathrm{N}^{\circ} 5$.

\section{B. Respecto a la metodología:}

Se empleó la metodología ACI 211.2 utilizando el método 2 (mezclas de prueba), que se ajusta a los tres materiales de peso ligero, obteniéndose una ecuación polinómica de segundo grado (Fig. 2) que relaciona el contenido de cemento en $\mathrm{kg} / \mathrm{m} 3$ de agregado, en función de la resistencia. Es decir, en la parte baja para un incremento significativo de resistencia se necesita un pequeño incremento en el contenido de cemento, en cambio para obtener altas resistencias se debe incrementar de manera considerable el contenido de cemento.

No es recomendable el uso de un contenido de cemento de $30 \mathrm{~kg} / \mathrm{m} 3$ (mínimo valor de la curva) para un bloque tipo $\mathrm{C}$, pues este mampuesto es muy frágil.

La cantidad de agua que debe colocarse no está limitada a la necesaria para lograr saturar el agregado y formar la pasta, sino a la adecuada para permitir formar el bloque sin segregación durante la vibro-compactación y sin fisuración por falta de humedad al iniciar el secado.

A pesar de estimar en laboratorio un valor de absorción para el agregado, no se puede comprobar su absorción real en el corto tiempo de mezclado, moldeo y luego durante el secado. Esto dificulta la estimación verdadera de la relación a/c empleada.

\section{Respecto a las características de los bloques:}

Los valores obtenidos de esfuerzo a compresión empleando la metodología ACI 211.2 con el método 1, llegan al 20 o $30 \%$ del valor esperado. Además, no fue posible colocar la cantidad total calculada de agua, pues dicha cantidad producía segregación durante la vibrocompactación e irregularidades al formar el bloque. Este exceso de agua puede deberse a que el método no estima adecuadamente la absorción del agregado liviano. Con esto se comprueba que esta metodología no es aplicable cuando se intenta emplear la fracción gruesa y fina provenientes de materiales de peso ligero.

La condición de humedad de los agregados empleados para la fabricación de bloques influye directamente en la resistencia a compresión. La cantidad total de agua en la mezcla, para el caso de agregados húmedos fue mayor que la cantidad total usada para el caso de agregados secos. Sin embargo, al usar agregados húmedos, tal como recomienda el ACI 211.2, se obtuvieron mejores resistencias. De esto se puede inferir que cuando se usaron agregados secos hubo agua que se absorbió posteriormente al mezclado y no pudo hidratar al cemento. Dicha absorción fue menor al agregado en condición húmeda.

Se logró obtener las dosificaciones, en peso y volumen, para elaborar bloques tipo A, B y C. Sin embargo, para el bloque tipo A se cumple únicamente con el requisito de resistencia mínima por bloque (TABLA IV), mas no el promedio de 3 unidades de acuerdo a lo que determina la norma. Esto debido a que un contenido de cemento mayor a $245 \mathrm{~kg} / \mathrm{m} 3$, no permite que la mezcla sea trabajable.

El bloque tipo B, llega satisfactoriamente a la resistencia establecida por la normativa. Este puede ser usado en paredes divisorias no soportantes, es decir, como mampostería no estructural.

El bloque tipo $\mathrm{C}$, llega satisfactoriamente a la resistencia establecida por la norma utilizando el límite inferior del 
contenido de cemento determinado en la curva. Su uso está destinado para alivianamiento de losas.

No se satisface el espesor mínimo de tabiques para el bloque tipo $\mathrm{A}$, esta es una variable que depende exclusivamente de las dimensiones del molde que se emplea en las fábricas de bloques artesanales, cuya modificación estaba fuera del alcance de esta investigación. Sin embargo, se mantuvo un área normalizada de tabiques mayor al que recomienda la norma.

La resistencia está ligada directamente a la densidad; como se evidencia en la Fig. 5, donde a menor densidad menor resistencia a compresión.

La densidad es inversamente proporcional a la absorción como se evidencia en la Fig. 4, donde a mayor absorción menor densidad.

Los bloques obtenidos, en todos los casos, se clasifican como livianos al tener una densidad menor a $1680 \mathrm{~kg} / \mathrm{m} 3$ (TABLA III).

Los bloques tipo A presentan un valor promedio de 230 $\mathrm{kg} / \mathrm{m} 3$ que es inferior al máximo permitido por la norma NTE INEN 3066 que es de $288 \mathrm{~kg} / \mathrm{m} 3$. Para los bloques tipo $\mathrm{B}$ y $\mathrm{C}$ no hay restricción de un valor máximo de absorción, sin embargo, se determinó que tienen un valor promedio de 270 y $350 \mathrm{~kg} / \mathrm{m} 3$ respectivamente.

\section{Respecto al costo:}

Se determinó que el costo directo del bloque artesanal sin la proporción optimizada es de 0.1543 dólares y su venta se estima en 0.18 dólares obteniéndose una utilidad del $17 \%$. La resistencia promedio a la que llega a los 28 días es de $33.4 \mathrm{~kg} / \mathrm{cm} 2$ clasificándolo como tipo $\mathrm{C}$, $\sin$ embargo, al optimizar la proporción de agregados $\mathrm{y}$ utilizando la misma cantidad de cemento, no hubo variación en el costo, pero se alcanzó un incremento del $25 \%$ en la resistencia.

La fabricación de un mampuesto tipo $\mathrm{B}$ con la proporción de agregados optimizada, reduce en un $9 \%$ el costo respecto del artesanal, debido a que además de hacer eficiente la proporción, se disminuye el contenido de cemento.

La fabricación de un mampuesto tipo $\mathrm{C}$ con la proporción de agregados optimizada, reduce en un $18 \%$ el costo respecto del artesanal.

Para elaborar un bloque tipo A (resistencia mínima por bloque) se debe aumentar la resistencia cuatro veces, por lo cual el incremento en el costo es de $128 \%$, esto considerando que se comparó con el costo de un bloque artesanal tipo C. Es importante mencionar que el beneficio de este tipo de bloque radica en que puede ser destinado a una pared estructural.

El número de bloques que se obtiene con una determinada cantidad de material varía debido a los desperdicios que se generan en las máquinas de mezclado y conformado. Aproximadamente, para un volumen de agregados de $0.42 \mathrm{~m} 3$ se obtendrán entre 60 y 70 bloques.

\section{RECONOCIMIENTOS}

Este trabajo ha sido financiado parcialmente por el Proyecto de Investigación PII17-11 y forma parte de la
Tesis Determinación de dosificación para elaborar bloques huecos de hormigón que cumplan con la actual norma INEN 3066.

Los autores desean expresar su agradecimiento a María Auxiliadora Naranjo, José Gabriel Merino Cabezas por su participación en el trabajo y al Laboratorio de Ensayo de Materiales, Mecánica de Suelos y Rocas (LEMSUR).

\section{REFERENCIAS}

[1] NCMA. (Nov. 2012). Concrete Masonry Designs. (M. Arntson-Terrell, Ed.) Pg. 23. Obtenido de http://www.ncmabr.org/pdfs/masterlibrary/CMD 2012 Nov Dec.pdf

[2] Bloques de Hormigón. Requisitos y Métodos de Ensayo, NTE INEN 3066, Quito, Ecuador, 2016.

[3] Áridos. Análisis granulométrico de los áridos, fino y grueso, NTE INEN 696, Quito, Ecuador, 2011.

[4] Áridos. Determinación de la densidad, densidad relativa (gravedad específica) y absorción del árido grueso, NTE INEN 857, Quito, Ecuador, 2010.

[5] Áridos. Determinación de la masa unitaria (Peso volumétrico) y el porcentaje de vacios, NTE INEN 858, Quito, Ecuador, 2010.

[6] Lightweight Aggregates for Structural Concrete, ASTMC330, Estados Unidos, 2017.

[7] Luna, G. V., Estudio del Hormigón: Guía didáctica para el diseño de mezclas, Quito, Ecuador: Edicumbre, 2014.

[8] Bonett Díaz, R. L., Vulnerabilidad y riesgo sísmico de edificios. Aplicación a entornos urbanos en zonas de amenaza alta y moderada, Barcelona, España: Tesis Doctoral, 2003.

[9] Áridos. Determinación de la densidad, densidad relativa (gravedad específica) y absorción del árido fino, NTE INEN 856, Quito, Ecuador, 2010 